\title{
Treatment Methods for Bone Metastasis-induced Pain
}

\author{
(1) Savaş ÇÖMLEK \\ Department of Anesthesiology and Reanimation, Gayrettepe Florence Nightingale Hospital, Istanbul-Turkey
}

\section{Introduction}

Bone metastasis-induced cancer pain is chronic pain with complex pathogenesis that affects daily activities and social life due to its physical, psychological, and emotional effects.[1] The most important cause of treatment limitations is the lack of information regarding the pain's mechanisms. According to our current knowledge metastatic bone pain is originating from inflammatory and neuropathic pathways, which could be focal, multifocal, or generalized. Among the neurochemical substances responsible for pain behavior in different forms, the most well known are substance $\mathrm{P}, \mathrm{c}$-Fos and dynorphin. Numerous studies have shown that tumor and inflammatory cells that secrete substances that cause sustained activation of osteoclasts cause bone cancer pain. Painful syndromes that also occur in nerve compression and injuries caused by tumor invasion and growth are well-known types in patients with bone metastasis.[2]

When bone metabolism changes in patients with bone metastases were examined, it was shown that pain was associated with bone destruction. The increase in osteclastic activity responsible for bone destruction is under the control of tumor necrosis factor-alpha (TNF-alpha) and other cytokines produced by cancer cells. The acidic environment in which bone destruction occurs is thought to cause pain by stimulating nociceptors. The severity and character of pain perception is also associated with changes in the dorsal root ganglia located in the spinal cord by stimulation of the nociceptors. [3]

One of the pain types impairing patients quality of life with bone metastasis-induced pain is breakthrough pain. Breakthrough pain is the transitory flare of pain in patients, which is continuous at a certain level and seen in about $75 \%$ of the patients.[4] Hyperalgesia, another important concept of pain, occurs due to central and peripheral sensitization induced by cancer. [5] Changes in the spinal cord caused by continuous peripheral stimulation, resulting in decreased pain threshold, which is known as the mechanism of central sensitization.[6] Reviewing the bone metastasis-induced pain management would be helpful by dividing it into three basic approaches, including pharmacological, interventional, and surgical.

\section{Pharmacological Approach}

Widely known for treating painful bone metastases, the pharmacological approach is based on the WHO analgesic ladder. Treatment usually starts with a nonsteroidal anti-inflammatory agent or paracetamol. The second step consists of a combination of weak opioids (codeine and tramadol) and non-opioid analgesics, while the third step consists of strong opioids. Consistent with the three-step analgesic guideline of the World Health Organization, while themainstay of managing cancer pain remains to be opioid use, this approach does not provide enough pain control in about a quarter of patients. As a consequence for this reason, interventional pain treatment is an valuable fourth step in controlling cancer related pain.[7,8] Thus, the generally accepted view is that an adjuvant agent may be added if the clinician deems appropriate at any step, or an interventional procedure may be performed.

\section{Non-steroidal Anti-inflammatory Drugs}

Non-steroidal anti-inflammatory drugs (NSAID) fall into the first step of the WHO analgesic ladder. While NSAIDs substantially reduce cancer-induced pain in a meta-analysis of twenty five randomized controlled tri- 
als on the use of NSAIDs to manage cancer pain, their use in the treatment of metastasis-induced bone pain remains controversial. The most critical concern is the negative and serious side effects of NSAIDs on the stomach and kidneys. In a Cochrane review of 42 clinical trials on the use of NSAIDs for the management of cancer pain, no conclusions could be drawn whether NSAID is superior or safer.[9] This agent's main mechanism is to inhibit the cyclooxygenase (COX) enzymes, which play a role in the synthesis of prostaglandins regulating various cellular functions, including pain perception. In tumor cells, COX-2 has increased activity. Therefore, reducing the COX activity would prevent pain perception.[10]

\section{Opioids}

Opioids are categorized as weak and strong and fall in the second and third steps of the WHO analgesic ladder. They are the useful and cost effective therapeutic group for the management of cancer pain. The analgesic effects of the opioids largely depend on $\mu$-receptor saturation. Nevertheless, the type and severity of the pain, previous opiate use and the personal differences in the receptor distribution are the other factors determining its efficacy. The main adverse effects of opioids include respiratory depression, addiction, physical dependence, tolerance, sedation, constipation, nausea, and vomiting.

Clinicians recommend two approaches to manage the analgesic effects of opioids. First, the best possible analgesic effect combined with the way to reduce side effects is individualized therapy based on the pharmacogenomics of the cancer type. Second, titration of the medication based on the pharmacodynamic and pharmacokinetic studies obtains the best performance from opioids using minimum dosages. As with the combination of sublingual fentanyl with other long-acting narcotics for rapid onset of effect on breakthrough pain, narcotic agents with different properties may be combined based on the pain character. Various medications are used to reduce the adverse effects, such as ondansetron and metoclopramide, laxatives, and methylphenidate. Opioid sensitization is an important issue causing reduced efficacy of the agent when used for a prolonged period. Therefore, treating the pain using increased doses of the same opioid may ineffective.[11]

\section{Bone-targeted Agents (Bisphosphonates and Deno- sumab)}

Bisphosphonates are used commonly for the treatment and prevention of bone metastasis induced pain and other complications. These medications effectively control cancer pain by improving the acidic microenvironment of the local tumor bone tissue and reducing bone resorption.[12] Due to both their effects in preventing metastasis-induced complications and analgesic effects, bisphosphonates are recommended as a part of the treatment regimen in patients followed for bone metastasis (evidence IIb).[13] Zoledronic acid is the drug from the first bisphosphonate group with proven effectiveness in preventing and delaying bone metastases in prostate cancer and renal cell cancer.[14]

Denosumab is a human monoclonal (IgG2) antibody developed against RANKL. Although studies are currently ongoing, its role in bone pain is promising, and it is used as an alternative to bisphosphonates. [12]

\section{Tricyclic Antidepressants}

Tricyclic antidepressants can be used for chronic pain in cancer patients due to their beneficial effects on their psychological status and sleep. Clinical studies have reported that they affect depressive symptoms and modulate the patient's perception of pain. Selective norepinephrine and serotonin reuptake inhibitors, including venlafaxine, paroxetine, duloxetine, and citalopram, have been proven to effectively treat neuropathic pain.[15]

\section{Corticosteroids}

Corticosteroids are often used as adjuvants, treatment of cancer-related pain syndromes. These include bone and neuropathic pain caused by the infiltration of neural structures or metastatic entrapment, headache caused by intracranial pressure increase, arthralgia, pain caused by persistent inflammation and compression on the surrounding tissues, and capsule pain caused by organ strain.[16] Nevertheless, when corticosteroids are used for a long time, they can cause side effects such as immunosuppression, hypertension, hyperglycemia, stomach ulcers and psychosis. The benefit-risk analysis in cancer patients indicated that steroid use benefits, especially central nervous system involvement, outweigh the risks.

\section{Radiotherapy}

Radiotherapy (RTx) is widely used in the treatment of cancer patients and is one of the most effective methods in terms of pain relief. The Radiation Therapy Oncology Group reported that $80-90 \%$ of patients experienced partial or complete pain relief 10-14 days after radiotherapy applied to bone metastases.[17] 
Radiotherapy, when combined with opioids, provides potent analgesic effects in localized metastatic bone pain. Evidence showed that low-dose, short-term radiotherapy cycles are as effective as the longer-term cycles.[18] Transient aggravation of pain may occur after palliative radiotherapy, and the use of prophylactic dexamethasone helps alleviate this aggravation.[13] Recently, studies recommended the combination of radiotherapy with radiofrequency and cementoplasty procedures, especially in vertebra metastases.[19-22]

\section{Radiopharmaceuticals}

The clinical development of bone selective radiopharmaceuticals is based on providing more effective antitumor activity while protecting the healthy tissues from the detrimental effects of the radiation by targeting the tumor-related skeletal regions and directing the moderate-to high particle radiation to the relevant site. Samarium-153-ethylene-diamine-tetra methylene phosphonic acid and strontium-89-chloride are radionuclides that can be used clinically, and their half-lives and radiation energies are different. For this reason, these products, which can be used in different indications considering their side effect potentials, are approved in the USA and Europe.[14]

\section{Interventional Methods}

The improvements in the survival rates of the patients due to the advancements in cancer therapy substantially increased the need for interventional methods of pain treatment. In bone metastases, the rate of patients with uncontrollable pain was reported to be $40 \%$. The most effective way to reduce this rate is through the availability of interventional pain treatment methods.[23]

Different opinions have been suggested to redefine the WHO analgesic ladder. One of them is related to the timing of the interventional procedures. There is increasing evidence showing that early blocking procedures without waiting for the pain to become severe or resistant are beneficial. Studies have shown improved outcomes, a substantial reduction in opioid consumption, improved quality of life in the patients undergoing early blocking procedures than later.[24-26]

The benefits of the early interventional methods should be considered in light of this information. When the conventional opioids based approaches are insufficient and ineffective, interventional techniques, including nerve blocks, neuroablative/neurodestruc- tive methods, intrathecal or epidural drug administration, should be evaluated and performed with a multidisciplinary approach.

\section{Neurodestruction}

It is based on preventing transmission of the pain pathways of pain signalization from the spinal cord to the brain using chemical, mechanical and thermal instruments. Neurodestruction can be administered on nerve fibers belonging to the localization of bone metastasis-induced pain, sparing the motor functions. Currently, many procedures performed using open surgical interventions in the past are performed percutaneously guided by imaging. Pain transmission can be blocked at all levels including nerve, nerve root, nerve root ganglion, spinal cord, thalamus or brainstem.

One of the techniques used for neurodestruction is the CT-guided percutaneous cordotomy procedure based on cutting the spinothalamic pathway, mostly at the cervical level. This technique causes loss of pain on the other side of the body, leading to pain relief. Other neurodestructive methods, thalamotomy, and cingulotomy, are irreversible invasive procedures rarely used due to their potential side effects, although they are known to be effective and beneficial.[15]

Injection of neurolytic agents to ganglia is an effective treatment method for chronic cancer pain complicated by bone metastases. Pancreatic cancer-induced chronic abdominal pain can be treated by celiac/ splanchnic plexus block, which is effective and safe in controlling the pain by providing pain-free status in $70-90 \%$ of abdominal cancer patients. [27] Most common side effects due to celiac plexus block consists of orthostatic hypotension, local pain and diarrhea that can be managed with early diagnosis and adequate conservative treatment. Superior Hypogastric plexus, ganglion impar block and neurolysis can be used in cancers causing pelvic pain.[28] However, superior hypogastric plexus block is a relatively less researched technique in the treatment of cancer-related pelvic pain, and it is mostly indicated late, which reduces its effectiveness. Therefore, it is thought to be less effective than the celiac plexus block. Nonetheless, the hypogastric block can be used in medically persistent pelvic pain cases, and to date, it has no known complication. As in the intercostal nerves, block and neurolysis applied to the affected peripheral nerves are effective pain treatment methods in appropriate cases in applications performed with alcohol and phenol. Vertebroplasty and kyphoplasty are other effective techniques that can be used as an adjuvant with radiotherapy for the treat- 
ment of pain caused by painful vertebral compression fractures in metastatic patients. [29,30]

The most well-known and effective application of radiofrequency ablation is the use of imaging-guided tumor ablation. Several publications showed the favorable results of bone cement injection and augmentation in the vertebral corpus and long bones and nonspinal structures.[19-21]

\section{Neuromodulation}

Electrical neuromodulation is based on the electrical stimulation of the peripheral nerve, or the dorsal column of the spinal cord and brain. Spinal cord stimulation (SCS) effectively controls mainly neuropathic pain in patients with arachnoiditis. However, its efficacy and use in the control of nociceptive pain is controversial. Several studies using spinal cord stimulation showed efficacy. According to one of them, it was shown that there was a $60 \%$ improvement in pain intensity and quality of life for as long as 3 years. However, it is not to be accepted as one of the first-choice treatment options for persistent chronic pain.[29]

\section{Intrathecal Port/Pump Placement}

An important tool in controlling severe cancer pain is the use of intrathecal drugs, which have been shown to be effective in many chronic pain syndromes. For this, For this, primarily opiates, local anesthetics, ziconitidine and some alpha agonists are widely used. These drugs can be administered alone or in combination for controlling persistent pain. These medications are administered mostly through automated pumps delivering the medication into the intrathecal space at a determined infusion rate based on the patient's need and the intensty of the pain. Intrathecal administration of the aforementioned analgesics causes less systemic side effects of these drugs. Compared to the other routes of administration (oral, IV, transdermal), intrathecal or epidural administrations provide substantially lower doses and higher efficacy rates. The secret to qualified and high efficacy is related to combining narcotics with local anesthetics, which cannot be used effectively through other routes. Intrathecal drug delivery systems are an effective method of treatment, which allows comprehensive management of the cancer pain affecting the whole body, proven in a multi-center, randomized clinical trial.[31] According to this trial, systemic adverse effects were reduced by $50 \%$ using infusion through an intrathecal pump. Thus, it showed a substantial reduction in the rates of fatigue and loss of consciousness and improved survival rates in patients with implantable intrathecal drug delivery systems. [32] This pump can be implanted into the abdominal subcutaneous area to provide continuous infusion. Today, pumps sold in the market are programmable devices and can be remotely managed via digital connections. The reservoirs of these pumps should be refilled at regular intervals. In addition to opioids, clonidine, bupivacaine, baclofen, and ziconotide can be administered intrathecally. Ziconotide, a novel agent, approved by FDA in 2004, acts through calcium channels in nerve tissue. It provides pain control through neurochemicals such as substance P, glutamate, and calcitonin gene-related peptide, which modulate nociceptive pain stimulation in the spinal cord.[33] Simple management of the intrathecal pumps and used medications and the reversibility of the possible adverse effects make the intrathecal pumps a commonly accepted treatment method for long-term severe cancer pain in patients due to bone metastases.[34] In cases where pump use is not possible for pain management, bolus administration using subcutaneous ports and external infusion systems can also be used.

\section{Epidural Port/Catheter Placement}

Epidural administrations are as effective as intrathecal ports and catheters in treating cancer pain. However, there is no randomized trial comparing the efficacy of drug infusions using both methods. The decision to use intrathecal or epidural drug delivery is based on the localization of the pain, catheterization technique, and patient\&\#39;s life expectancy. For example, in intrathecal space compression, epidural catheterization may be preferred over intrathecal administration for optimal neuraxial spread. In patients without long life expectancy, the catheter might be tunneled subcutaneously and/or connected to an external subcutaneous port accessed per-cutaneously. In some cases, intrathecal administration is preferred due to catheter durability and the need for lower doses. However, some patients prefer epidural tunneled intermittent catheters as they are less invasive and inexpensive.

\section{Surgery}

The treatment goals in patients with pathological fractures due to bone metastases include pain relief, morbidity reduction, and increasing the functions and skeletal integrity of the patients to an optimal level for the remaining part of their lives. These patients may undergo surgical therapies using internal fixation and 
instrumentation to provide palliative care in better conditions. In patients with epidural/spinal cord compression or mechanic instability caused by vertebral metastasis, surgical options should be considered.

\section{References}

1. Zhu XC, Zhang JL, Ge CT, Yu YY, Wang P, Yuan TF, et al. Advances in cancer pain from bone metastasis. Drug Des Devel Ther 2015; 9:4239-45.

2. Mantyh PW, Clohisy DR, Koltzenburg M, Hunt SP. Molecular mechanisms of cancer pain. Nat Rev Cancer 2002;2(3):201-9.

3. Honore P, Luger NM, Sabino MA, Schwei MJ, Rogers SD, Mach DB, et al. Osteoprotegerin blocks bone cancer-induced skeletal destruction, skeletal pain and pain-related neurochemical reorganization of the spinal cord. Nat Med 2000;6(5):521-8.

4. Smith HS, Mohsin I. Painful boney metastases. Korean J Pain 2013;26(3):223-41.

5. Peng X, Guo W, Ren T, Lou Z, Lu X, Zhang S, et al. Differential expression of the RANKL/RANK/OPG system is associated with bone metastasis in human nonsmall cell lung cancer. PLoS One 2013;8(3):e58361.

6. Luger NM, Honore P, Sabino MA, Schwei MJ, Rogers SD, Mach DB, et al. Osteoprotegerin diminishes advanced bone cancer pain. Cancer Res 2001;61(10):4038-47.

7. Swarm RA, Abernethy AP, Anghelescu DL, Benedetti C, Buga S, Cleeland C, et al. Adult cancer pain. J Natl Compr Canc Netw 2013;11(8):992-1022.

8. Plancarte R, Hernández Porras C. Interventional pain management in cancer patients. Postgrad Med 2020;132(sup3):3-4.

9. McNicol E, Strassels SA, Goudas L, Lau J, Carr DB. NSAIDS or paracetamol, alone or combined with opioids, for cancer pain. Cochrane Database Syst Rev 2005;(1):CD005180.

10. Sabino MA, Ghilardi JR, Jongen JL, Keyser CP, Luger NM, Mach DB, et al. Simultaneous reduction in cancer pain, bone destruction, and tumor growth by selective inhibition of cyclooxygenase-2. Cancer Res 2002;62(24):7343-9.

11. Ballantyne JC, Mao J. Opioid therapy for chronic pain. N Engl J Med 2003;349(20):1943-53.

12. Walker K, Medhurst SJ, Kidd BL, Glatt M, Bowes M, Patel S, et al. Disease modifying and anti-nociceptive effects of the bisphosphonate, zoledronic acid in a model of bone cancer pain. Pain 2002;100(3):219-29.

13. Chow E, Loblaw A, Harris K, Doyle M, Goh P, Chiu H, et al. Dexamethasone for the prophylaxis of radiationinduced pain flare after palliative radiotherapy for bone metastases: a pilot study. Support Care Cancer 2007;15(6):643-7.

14. Fitzgibbon DR, Loeser JD. Spine and Bone Pain. In: Fitzgibbon DR, Loeser JD, editors. Cancer Pain: Assessment, Diagnosis, and Management. Philadelphia: Williams\&amp;Wilkins; 2010. p. 303-13.

15. Ahmad I, Ahmed MM, Ahsraf MF, Naeem A, Tasleem A, Ahmed M, et al. Pain management in metastatic bone disease: a literature review. Cureus 2018;10(9):e3286.

16. Greenberg HS, Kim JH, Posner JB. Epidural spinal cord compression from metastatic tumor: results with a new treatment protocol. Ann Neurol 1980;8(4):361-6.

17. Tong D, Gillick L, Hendrickson FR. The palliation of symptomatic osseous metastases: final results of the Study by the Radiation Therapy Oncology Group. Cancer 1982;50(5):893-9.

18. von Moos R, Costa L, Ripamonti CI, Niepel D, Santini D. Improving quality of life in patients with advanced cancer: targeting metastatic bone pain. Eur J Cancer 2017;71:80-94.

19. Plancarte R, Guajardo J, Meneses-Garcia A, Hernandez-Porras C, Chejne-Gomez F, Medina-Santillan R, et al. Clinical benefits of femoroplasty: a nonsurgical alternative for the management of femoral metastases. Pain Physician 2014;17(3):227-34.

20. Cazzato RL, Palussière J, Buy X, Denaro V, Santini D, Tonini G, et al. Percutaneous long bone cementoplasty for palliation of malignant lesions of the limbs: a systematic review. Cardiovasc Intervent Radiol 2015;38(6):1563-72.

21. Kurita GP, Sjøgren P, Klepstad P, Mercadante S. Interventional techniques to management of cancer-related pain: clinical and critical aspects. Cancers (Basel) 2019;11(4):443.

22. Fahim DK, Ragheb A, Vanood A. The addition of radiofrequency tumor ablation to vertebroplasty reduces local recurrence in spinal metastases from breast cancer. Neurosurgery 2020;67(Supplement 1):Abstract 682.

23. Meuser T, Pietruck C, Radbruch L, Stute P, Lehmann KA, Grond S. Symptoms during cancer pain treatment following WHO-guidelines: a longitudinal follow-up study of symptom prevalence, severity and etiology. Pain 2001;93(3):247-57.

24. Zhong W, Yu Z, Zeng JX, Lin Y, Yu T, Min XH, et al. Celiac plexus block for treatment of pain associated with pancreatic cancer: a meta-analysis. Pain Pract 2014;14(1):43-51.

25. Amr YM, Makharita MY. Neurolytic sympathectomy in the management of cancer pain-time effect: a prospective, randomized multicenter study. J Pain Symptom Manage 2014;48(5):944-56.e2.

26. Wong GY, Schroeder DR, Carns PE, Wilson JL, Martin DP, Kinney MO, et al. Effect of neurolytic celiac plexus 
block on pain relief, quality of life, and survival in patients with unresectable pancreatic cancer: a randomized controlled trial. JAMA 2004;291(9):1092-9.

27. Eisenberg E, Carr DB, Chalmers TC. Neurolytic celiac plexus block for treatment of cancer pain: a meta-analysis. Anesth Analg 1995;80(2):290-5.

28. de Leon-Casasola OA, Kent E, Lema MJ. Neurolytic superior hypogastric plexus block for chronic pelvic pain associated with cancer. Pain 1993;54(2):145-51.

29. Miguel R. Interventional treatment of cancer pain: the fourth step in the World Health Organization analgesic ladder? Cancer Control 2000;7(2):149-56.

30. Fourney DR, Schomer DF, Nader R, Chlan-Fourney J, Suki D, Ahrar K, et al. Percutaneous vertebroplasty and kyphoplasty for painful vertebral body fractures in cancer patients J Neurosurg 2003;98(1 Suppl):21-30.
31. Smith TJ, Staats PS, Deer T, Stearns LJ, Rauck RL, Boortz-Marx RL, et al. Randomized clinical trial of an implantable drug delivery system compared with comprehensive medical management for refractory cancer pain: impact on pain, drugrelated toxicity, and survival. J Clin Oncol 2002;20(19):4040-9.

32. Gilmer-Hill HS, Boggan JE, Smith KA, Wagner FC Jr. Intrathecal morphine delivered via subcutaneous pump for intractable cancer pain: a review of the literature. Surg Neurol 1999;51(1):12-5.

33. Hassenbusch SJ, Gunes S, Wachsman S, Willis KD. Intrathecal clonidine in the treatment of intractable pain: a phase I/II study. Pain Med 2002;3(2):85-91.

34. Gatscher S, Becker R, Uhle E, Bertalanffy H. Combined intrathecal baclofen and morphine infusion for the treatment of spasticity related pain and central deafferentiation pain. Acta Neurochir Suppl 2002;79:75-6. 